\title{
A NOVEL HYBRID AUTOMATON FRAMEWORK FOR MULTI-PHASE EPIDEMIC MODELLING
}

\author{
Eva M. Navarro-López \\ Nurdan Çabukoğlu \\ School of Mathematics \\ and Computer Science \\ University of Wolverhampton \\ Alan Turing Building \\ Wulfruna St, Wolverhampton WV1 1LY, UK \\ \{E.NavarroLopez,N.Cabukoglu\}@wlv.ac.uk
}

\begin{abstract}
A framework for the multi-phase epidemic modelling of SEIARD (Susceptible-Exposed-symptomatic Infectious-Asymptomatic infectious-Recovered by immunity or by vaccination-Dead due to the disease) subpopulations is produced with switching transmission rate, basic reproduction ratio and vaccination strategy. The key novel feature of our model is that we reproduce the different phases of the evolution of the infectious disease by using a hybrid automaton with different discrete locations corresponding to each of the phases of the disease. This is a general modelling framework applicable to the spreading of infectious diseases. We show how the proposed model works with the simulation of different scenarios.
\end{abstract}

Keywords: epidemics, hybrid automata, switching disease phases, dynamical models.

\section{INTRODUCTION}

There has been a surge of epidemic models since COVID-19 struck at the end of 2019 (Graphext 2021). These models are typically based on the classical epidemic SIR, SEIR or SEIRD models, where $S, E, I, R$ and $D$ stand for individuals who are susceptible, exposed, infected, recovered and dead due to the disease, respectively. Few models have differentiated symptomatic $(I)$ and asymptomatic $(A)$ infected subpopulations (De la Sen, Ibeas, Alonso-Quesada, and Nistal 2017, De la Sen, Agarwal, Nistal, Alonso-Quesada, and Ibeas 2018). Recent models have considered switching transmission rates (Chladná, Kopfová, Rachinskii, and Rouf 2020, Liu and Stechlinski 2017, Pröll 2013), under the theory of discontinuous and switched dynamical systems. There are few models that consider vaccination strategies (Meng and Chen 2008, De la Sen, Agarwal, Nistal, Alonso-Quesada, and Ibeas 2018).

Surprisingly, as far as we are aware, it is still a challenge to reproduce the dynamical evolution over time of the phases of an infectious disease; these phases corresponding to different contention, confinement or vaccination measures. The model proposed in this paper offers a solution to this problem and takes one step further to produce less simplistic epidemic models for the complex dynamical behaviours involved in the spreading of infectious diseases over different evolution phases. A novel framework based on the hybrid 
automaton abstraction is used (Navarro-López and Carter 2011, Navarro-López and Laila 2013, NavarroLópez and Carter 2016). Hybrid automata are a useful model of hybrid dynamical systems, since they explicitly show the interaction between the continuous and the discrete parts of the system. They are computational dynamical models that specify in an elegant way the transitions and event-triggered phenomena of systems with multiple types of discontinuities and switching dynamical behaviours.

One of the key aspects of the novel hybrid automaton-based framework proposed here is its modularity, since any pre-existing epidemic model can be used in each discrete location. We consider a switching transmission rate $\beta$, and consequently, a varying basic reproduction ratio $R_{0}$. Additionally, we consider a switching vaccination strategy $(\lambda)$. Each different value of $\beta, \lambda$ and $R_{0}$ corresponds to a different phase of the evolution of the disease. The switch between the different phases of the disease will depend on dynamical transitions or conditions on the dynamical states of the system, which are the time-dependent probabilities to belong to the $S, E, I, A, R$ and $D$ subpopulations. The result is what we call the SEIARD hybrid automaton. For the dynamics within each discrete location of the SEIARD hybrid automaton, we propose a new SEIARD epidemic model that combines some of the proposals made in the works of (De la Sen, Ibeas, Alonso-Quesada, and Nistal 2017, De la Sen, Agarwal, Nistal, Alonso-Quesada, and Ibeas 2018, Meng and Chen 2008). The model uniquely unifies the distinction between symptomatic and asymptomatic infectious subpopulations, the consideration of an immunity period, and the changes of the transmission rate and the vaccination strategy depending on the evolution of the disease.

These results are significant because we offer an elegant and modular modelling framework to further our understanding of the dynamical evolution of infectious diseases. Moreover, we propose an extended version of the SEIARD epidemic model. We are aware of the limitations of our proposal in order to reproduce real scenarios in concrete diseases. However, the goal of this paper is to propose a general modelling framework to better understand the complexities of the spreading of diseases that other models cannot reproduce, including the design of the transmission rate and vaccination strategy as control inputs, which may be used to make recommendations to health or government agencies.

The rest of the paper is organised as follows. Section 2 describes the basic SEIARD dynamical model for each phase of the disease $i$. Additionally, we calculate the basic reproduction ratio $R_{0_{i}}$, which reflects the reproductive power of the disease, associated with the new SEIARD model proposed for each phase of the disease. The SEIARD hybrid automaton is presented in Section 3. Simulations of the SEIARD dynamical model and the hybrid automaton are given in Section 4. Conclusions are given in the last section. The simulations presented in this paper have been done with MATLAB and OpenModelica.

\section{SEIARD MODEL FOR EACH PHASE OF THE DISEASE}

\subsection{Dynamical evolution of the subpopulations in each phase of the disease}

In this section, we propose the model for each phase $i$ of the disease, with $i \in\{1,2, \ldots, M\}$, and $M$ the number of phases considered in our epidemic model. Our model is a modified version of the SEIARD epidemic disease model proposed in (De la Sen, Ibeas, Alonso-Quesada, and Nistal 2017, De la Sen, Agarwal, Nistal, Alonso-Quesada, and Ibeas 2018), where the following subpopulations are considered: 1) susceptible $(S)$, exposed $(E)$, symptomatic infectious $(I)$, asymptomatic infectious $(A)$, recovered by immunity or vaccination $(R)$ and dead $(D)$. In our case, we do not consider that the dead people are infective. Figure 1 shows the rationale of our model. Additionally, we consider switching values for the transmission rates from the susceptible to the symptomatic infectious $\left(\beta_{i}\right)$ and from the susceptible to the asymptomatic infectious $\left(\beta_{A_{i}}\right)$ per each disease phase $i$. The proportion of susceptible individuals who have been vaccinated $\left(\lambda_{i}\right)$ is also different in each phase $i$, and $0 \leq \lambda_{i} \leq 1, \forall i \in\{1,2, \ldots, M\}$. The equations for what we call the epidemic SEIARD model for each phase $i$ of the disease are the following ones: 


$$
\begin{aligned}
\dot{S}(t) & =\tau-\left[\mu+\beta_{i} I(t)+\beta_{A_{i}} A(t)\right] S(t)+\eta R(t)-\lambda_{i} S(t), \\
\dot{E}(t) & =\left[\beta_{i} I(t)+\beta_{A_{i}} A(t)\right] S(t)-(\mu+\varepsilon) E(t), \\
\dot{I}(t) & =\varepsilon p E(t)-(\gamma+\mu+\alpha) I(t), \\
\dot{A}(t) & =\varepsilon(1-p) E(t)-(\gamma+\mu) A(t), \\
\dot{R}(t) & =\gamma[I(t)+A(t)]+\lambda_{i} S(t)-(\mu+\eta) R(t), \\
\dot{D}(t) & =\mu[I(t)+A(t)]+\alpha I(t),
\end{aligned}
$$

where $S(t)+E(t)+I(t)+A(t)+R(t)+D(t)=1$, for all time $t \geq 0$ and $S, E, I, A, R, D \in \mathbb{R}_{\geq 0}$, where $\mathbb{R}_{\geq 0}=$ $\{y \in \mathbb{R}: y \geq 0\}$. The dot denotes derivative with respect to time $t$. The state variables $S, E, I, A, R, D$ and the parameters are explained in Tables 1 and 2 . All the parameters in Table 2 belong to $\mathbb{R}_{+}$, where $\mathbb{R}_{+}=\{y \in$ $\mathbb{R}: y>0\}$, except $0 \leq \lambda_{i} \leq 1$. The following assumptions are considered:

1. $\tau=\mu$, that is, the birth rate in the susceptible population is the same as the natural death rate.

2. $\mu<<\gamma$. That is, the natural death rate cannot be greater than the recovery rate of infectious (both symptomatic and asymptomatic).

3. $\beta_{i}=\beta_{A_{i}}$ for all $i \in\{1,2, \ldots, M\}$, that is, the transmission rate from the susceptible to the symptomatic infectious is the same as the transmission rate from the susceptible to the asymptomatic infectious.

4. As in (De la Sen, Agarwal, Nistal, Alonso-Quesada, and Ibeas 2018), we assume that the mortality rate of the asymptomatic subpopulation is similar to the mortality rate of healthy people. That is, asymptomatic infectious individuals do not die from the disease, they can only die from natural death. However, there is an extra mortality rate associated with the symptomatic infectious subpopulation (expressed with parameter $\alpha$ ).

5. The dead bodies are not infectious.

6. We have a constant (closed) population size, $N$, and $s(t)+e(t)+i(t)+a(t)+r(t)+d(t)=N$ for all $t \geq 0$, with $s, e, i, a, r, d$ the number of individuals in each subpopulation. We define $S(t)=s(t) / N$, $E(t)=e(t) / N, I(t)=i(t) / N, A(t)=a(t) / N, R(t)=r(t) / N$ and $D(t)=d(t) / N$. Since $S(t)+E(t)+$ $I(t)+A(t)+R(t)+D(t)=1$ holds for all $t \geq 0$, then without loss of generality, we can assume that $S(t), E(t), I(t), A(t), R(t), D(t)$ is a system of time-dependent probabilities.

Table 1: State variables that dynamically evolve over time used in the SEIARD model

\begin{tabular}{cl}
\hline \hline State variable & Description \\
\hline S & Time-dependent probability to belong to the susceptible subpopulation \\
E & Time-dependent probability to belong to the exposed subpopulation \\
I & Time-dependent probability to belong to the symptomatic infectious subpopulation \\
A & Time-dependent probability to belong to the asymptomatic infectious subpopulation \\
R & Time-dependent probability to belong to the recovered by immunity/vaccination \\
& subpopulation \\
D & Time-dependent probability to belong to the dead subpopulation due to the disease \\
\hline
\end{tabular}

\subsection{Computation of the basic reproduction ratio $R_{0}$ for each phase of the disease}

The Jacobian method can be used to find $R_{0}$ for SEIR models. However, for more complex compartmental models like our SEIARD model, this method is hard to apply since it relies on the algebraic Routh-Hurwitz 


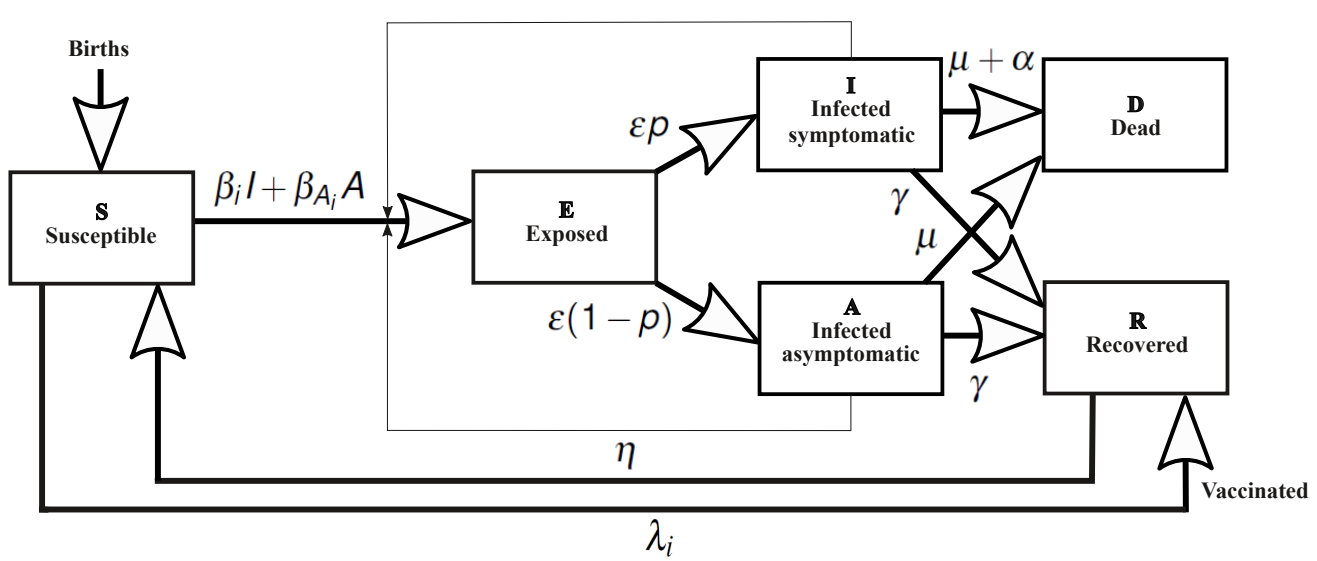

Figure 1: Relationships between subpopulations in the SEIARD epidemic model (1)-(6) for each phase of the disease. The thick arrows correspond to the transitions between subpopulations induced by the disease. The thin arrows correspond to coupled dynamics considered in the model that are induced by the disease. The natural deaths in all the subpopulations are not shown, only the disease-induced deaths are shown.

conditions for stability of the Jacobian matrix (van den Driessche 2017). Alternatively, we will use the method proposed in (Diekmann, Heesterbeek, and Metz 1990) and (den Driessche and Watmough 2002) that uses the next generation matrix. We denote the basic reproduction ratio for each phase of the disease as $R_{0_{i}}$.

Let $x=\left(x_{1}, x_{2}, \ldots, x_{n}\right)^{T}$ be the number of individuals in each compartment, where the first $m<n$ compartments contain infected individuals in the SEIARD model. Consider that the main model can be written in the form $\dot{x}_{i}=F_{i}(x)-V_{i}(x)$ for $i=1,2, \ldots, m$. In this splitting, $F_{i}(x)$ is the rate of appearance of new infections in compartment $i$, and $V_{i}(x)$ is the rate of other transitions between compartment $i$ and the other infected compartments. Now, we can define $F=\frac{\partial F_{i}\left(x_{0}\right)}{\partial x_{j}}$ and $V=\frac{\partial V_{i}\left(x_{0}\right)}{\partial x_{j}}$ for $1 \leq i, j \leq m$. Here, $F$ is entrywise non-negative and $V$ is a non-singular M-matrix, consequently, $V^{-1}$ is entrywise non-negative (Berman and Plemmons 1994). Let $\psi(0)$ be the number of initially infected individuals, therefore $F V^{-1} \psi(0)$ is an entrywise non-negative vector. This can give us the expected number of new infections. Thus, $F V^{-1}$ is the next generation matrix (van den Driessche 2017).

The next generation matrix approach is now applied to the SEIARD model (1)-(6). The infected compartments are $E, I, A$ and $D$. However, $D$ does not affect other infected terms. Therefore, we can use $E, I, A$ to define the matrices $F$ and $V$ for the SEIARD model:

$$
F=\left[\begin{array}{ccc}
0 & \beta_{i} S_{0} & \beta_{A_{i}} S_{0} \\
0 & 0 & 0 \\
0 & 0 & 0
\end{array}\right], \quad V=\left[\begin{array}{ccc}
\mu+\varepsilon & -\beta_{i} S_{0} & -\beta_{A_{i}} S_{0} \\
-\varepsilon p & \gamma+\mu+\alpha & 0 \\
-\varepsilon(1-p) & 0 & \gamma+\mu
\end{array}\right]
$$

Here $\beta_{i} S_{0}$ and $\beta_{A_{i}} S_{0}$ are the symptomatic and asymptomatic infection rates in a population of $S_{0}$ susceptible. We can find $V^{-1}$ by using the determinant method $V^{-1}=\frac{1}{\operatorname{det} V} \operatorname{adj}(V)$. Considering the third row of matrix $V$, the determinant is:

$$
\operatorname{det} V=-\varepsilon(1-p)(\gamma+\mu+\alpha) \beta_{A_{i}} S_{0}+(\gamma+\mu)\left[(\mu+\varepsilon)(\gamma+\mu+\alpha)-\varepsilon p \beta_{i} S_{0}\right]
$$


Table 2: Parameters used in the SEIARD model

\begin{tabular}{|c|c|c|}
\hline Parameter $\mathrm{D}$ & Description & Unit \\
\hline$R_{0_{i}}$ & $\begin{array}{l}\text { Basic reproduction ratio (reproductive power of the disease) } \\
\text { for phase } i \text { of the disease }\end{array}$ & Dimensionless \\
\hline$\beta_{i}$ & $\begin{array}{l}\text { Transmission, contact or infection rate from the susceptible to } \\
\text { the symptomatic infectious for phase } i \text { of the disease }\end{array}$ & day $^{-1}$ \\
\hline$\beta_{A_{i}}$ & $\begin{array}{l}\text { Transmission, contact or infection rate from the susceptible to } \\
\text { the asymptomatic infectious for phase } i \text { of the disease }\end{array}$ & day $^{-1}$ \\
\hline$\tau$ & "Birth" rate of susceptibles & day $^{-1}$ \\
\hline $\begin{array}{l}N \\
d e\end{array}$ & $\begin{array}{l}\text { Natural death rate ( } 1 / \mu \text { is an average natural life span of individuals), } \\
\text { deaths that are not caused by the disease are considered }\end{array}$ & day $^{-1}$ \\
\hline$\gamma$ & $\begin{array}{l}\text { Recovery (natural immune response) rate of infectious } \\
\text { (both symptomatic and asymptomatic), } 1 / \gamma \text { is the infectious period }\end{array}$ & day $^{-1}$ \\
\hline $\begin{array}{l}\mathrm{T} \\
\text { (b } \\
\text { fo }\end{array}$ & $\begin{array}{l}\text { Transition rate from the exposed to all infectious } \\
\text { (both symptomatic and asymptomatic), } 1 / \varepsilon \text { is the incubation period } \\
\text { for both symptomatic and asymptomatic infectious }\end{array}$ & day $^{-1}$ \\
\hline$\alpha$ & $\begin{array}{l}\text { Virus-induced average extra fatality rate associated with } \\
\text { symptomatic infectious }\end{array}$ & day $^{-1}$ \\
\hline$p$ & Fraction of the exposed individuals that become symptomatic infectious & Dimensionless \\
\hline $1-p$ & Fraction of the exposed individuals that become asymptomatic infectious & Dimensionless \\
\hline $\begin{array}{l}\mathrm{P} \\
\text { it }\end{array}$ & $\begin{array}{l}\text { Parameter such that } 1 / \eta \text { is the average duration of the immunity period, } \\
\text { it reflects the transition from the recovered to the susceptible }\end{array}$ & day $^{-1}$ \\
\hline$\lambda_{i}$ & Proportion of susceptible individuals vaccinated successfully & Dimensionless \\
\hline \multicolumn{3}{|c|}{$\left.\begin{array}{c}\varepsilon(1-p)(\gamma+\mu+\alpha) \\
\beta_{i} S_{0} \varepsilon(1-p) \\
(\mu+\varepsilon)(\gamma+\mu+\alpha)-\varepsilon p \beta_{i} S_{0}\end{array}\right]$} \\
\hline
\end{tabular}

Let us denote the elements $(i, j)$ of matrix $v$ by $v_{i j}$, then we can write:

$$
\begin{gathered}
F V^{-1}=\frac{1}{\operatorname{det} V}\left[\begin{array}{ccc}
\beta_{i} S_{0} v_{21}+\beta_{A_{i}} S_{0} v_{31} & \beta_{i} S_{0} v_{22}+\beta_{A_{i}} S_{0} v_{32} & \beta_{i} S_{0} v_{23}+\beta_{A_{i}} S_{0} v_{33} \\
0 & 0 & 0 \\
0 & 0 & 0
\end{array}\right] \\
\beta_{i} S_{0} v_{21}+\beta_{A_{i}} S_{0} v_{31}=\beta_{i}^{2} S_{0}^{2}(\gamma+\mu)+\beta_{A_{i}}^{2} S_{0}^{2}(\gamma+\mu+\alpha)=F V_{1}^{-1} \\
\beta_{i} S_{0} v_{22}+\beta_{A_{i}} S_{0} v_{32}=\beta_{i} S_{0}\left[(\mu+\varepsilon)(\gamma+\mu)-\varepsilon(1-p) \beta_{A_{i}} S_{0}\right]+\beta_{A_{i}}^{2} S_{0}^{2} \varepsilon p=F V_{2}^{-1} \\
\beta_{i} S_{0} v_{23}+\beta_{A_{i}} S_{0} v_{33}=\beta_{i}^{2} S_{0}^{2} \varepsilon(1-p)+\beta_{A_{i}} S_{0}\left[(\mu+\varepsilon)(\gamma+\mu+\alpha)-\varepsilon p \beta_{i} S_{0}\right]=F V_{3}^{-1}
\end{gathered}
$$

Suppose that individuals in $E$ are mildly infectious at a reduced rate $\zeta_{1} \beta_{i} S E$ and $\zeta_{2} \beta_{A_{i}} S E$ for $0<\zeta_{1}, \zeta_{2}<1$. Finally, $R_{0_{i}}$ can be defined as the summation of equations (10), (11) and (12) divided by $\operatorname{det} V$ : 


$$
R_{0_{i}}=\frac{1}{\operatorname{det} V}\left(F V_{1}^{-1}+\zeta_{1} F V_{2}^{-1}+\zeta_{2} F V_{3}^{-1}\right)
$$

\section{HYBRID AUTOMATON FOR THE MULTI-PHASE EPIDEMIC MODEL}

Let us define the state vector $x \in \mathbb{R}^{6}$ as $x=(S, E, I, A, R, D)^{T}$, and consider $\mathscr{X} \subseteq \mathbb{R}^{6}$ such that:

$$
\mathscr{X}=\left\{x \in \mathbb{R}_{\geq 0}^{6}: S+E+I+A+R+D=1\right\} .
$$

The system of equations (1)-(6) can be written as $\dot{x}=f_{i}(x)$, with $f_{i}: \mathscr{X} \rightarrow \mathscr{X}, i \in\{1,2, \ldots, M\}$, and $M$ the number of phases considered in our epidemic model.

\subsection{Dynamical considerations for the multi-phase epidemic model}

Now, we will reproduce the evolution of the SEIARD epidemic model defined in (1)-(6) in different stages of the transmission of the disease. Each of these phases is defined by a different transmission rate $\left(\beta_{i}\right)$ (for both symptomatic and asymptomatic infectious) and a different proportion of susceptible individuals vaccinated $\left(\lambda_{i}\right)$. Three key disease's phases are considered:

1. Free movement. The epidemic is under control or non-existent.

2. Uncontrolled spreading. The spreading of the disease increases, but there are not measures in place.

3. Confinement. There are hard restrictions to the movement of individuals to stop the transmission of the disease.

Additionally, we consider a group of different phases of deconfinement where there are relaxed restrictions to the movement of individuals from the phase of "Confinement" to the phase of "Free movement". We consider $P$ as the number of deconfinement phases between "Confinement" and "Free movement". Consequently, the number of total phases in our epidemic model is $M=P+3$. For the sake of simplicity, let us assume that $P=1$, and we will study the case of having four phases $(M=4)$ : Free movement, Uncontrolled spreading, Confinement and Deconfinement.

In each phase $i \in\{1, \ldots, M\}$, model (1)-(6) with a different transmission rate $\beta_{i}$ and a different $\lambda_{i}$ is considered. Let us define the conditions for transitions between these phases:

- From "Free movement", we may:

- Stay within "Free movement" if the number of infected individuals (both symptomatic and asymptomatic) decreases or is maintained constant, which is equivalent to say, if $\dot{I} \leq 0$ and $\dot{A} \leq 0$. By using equations (3) and (4), the following set can be defined:

$$
S_{1}=\left\{x \in \mathscr{X}: \quad I \geq \frac{\varepsilon p}{\gamma+\mu+\alpha} E, \quad A \geq \frac{\varepsilon(1-p)}{\gamma+\mu} E\right\} .
$$

- Switch to "Uncontrolled spreading" if the number of infected (symptomatic or asymptomatic) individuals increases, which is equivalent to say, if $\dot{I}>0$ or $\dot{A}>0$. By using equations (3) and (4), the following set can be defined:

$$
S_{2}=\left\{x \in \mathscr{X}: \quad I<\frac{\varepsilon p}{\gamma+\mu+\alpha} E\right\} \quad \bigcup\left\{x \in \mathscr{X}: \quad A<\frac{\varepsilon(1-p)}{\gamma+\mu} E\right\} .
$$


- Switch to "Confinement" if the number of exposed individuals is greater than the number of susceptible individuals, which is equivalent to say, if $E>S$.

- From "Uncontrolled spreading", we may:

- Stay within "Uncontrolled spreading" if the number of infected (symptomatic or asymptomatic) individuals keeps on increasing, that is, when $x \in S_{2}$.

- Switch to "Free movement" if the number of infected (both symptomatic and asymptomatic) individuals decreases or is maintained constant, that is, when $x \in S_{1}$.

- Switch to "Confinement" if the number of exposed individuals is greater than the number of susceptible individuals, which is equivalent to say, if $E>S$.

- Switch to "Deconfinement" if the number of exposed individuals decreases or is maintained constant, and consequently, if $\dot{E} \leq 0$. By using equation (2), the following set can be defined:

$$
S_{4}=\left\{x \in \mathscr{X}: \quad E \geq \frac{\beta_{i}}{\mu+\varepsilon}(I+A) S\right\} .
$$

Additionally to switch to "Deconfinement", we consider that the number of recovered individuals increases, that is, if $\dot{R}>0$. By using equation (5), the following set can be defined:

$$
S_{4}^{*}=\left\{x \in \mathscr{X}: \quad R<\frac{\gamma(I+A)+\lambda_{i} S}{\mu+\eta}\right\} .
$$

Moreover, we add two conditions: $I+A>\Delta$ and $D \leq \Delta_{2}$ with $\Delta$ and $\Delta_{2}$ two strictly positive constants. Consequently, the switch from "Uncontrolled spreading" to "Deconfinement" is produced if $x \in S_{4} \cap S_{4}^{*} \bigcap\left\{x \in \mathscr{X}: D \leq \Delta_{2}, I+A>\Delta\right\}$.

- From "Confinement", we may:

- Stay within "Confinement" if the number of exposed individuals increases or the number of recovered individuals decreases or is maintained constant. Consequently, if $\dot{E}>0$ or $\dot{R} \leq 0$. By using equation (2) and $\dot{E}>0$, the following set can be defined:

$$
S_{3}=\left\{x \in \mathscr{X}: \quad E<\frac{\beta_{i}}{\mu+\varepsilon}(I+A) S\right\} .
$$

Moreover, by using equation (5) and $\dot{R} \leq 0$, the following set can be defined:

$$
S_{3}^{*}=\left\{x \in \mathscr{X}: \quad R \geq \frac{\gamma(I+A)+\lambda_{i} S}{\mu+\eta}\right\} .
$$

Additionally, we consider that the number of dead people are big enough to be kept in "Confinement", which can be expressed as, $D>\Delta_{2}$. Consequently, the condition to stay within "Confinement" is $x \in S_{3} \bigcup S_{3}^{*} \cup\left\{x \in \mathscr{X}: D>\Delta_{2}\right\}$.

- Switch to "Deconfinement" if $x \in S_{4} \bigcap S_{4}^{*} \bigcap\left\{x \in \mathscr{X}: D \leq \Delta_{2}, I+A>\Delta\right\}$.

- From "Deconfinement", we may:

- Stay within "Deconfinement" if $x \in S_{4} \bigcap S_{4}^{*} \bigcap\left\{x \in \mathscr{X}: D \leq \Delta_{2}, I+A>\Delta\right\}$.

- Switch to "Confinement" if $x \in S_{3} \bigcup S_{3}^{*} \bigcup\left\{x \in \mathscr{X}: D>\Delta_{2}\right\}$.

- Switch to "Free movement" if the number of infected (both symptomatic and asymptomatic) individuals decreases or is maintained constant, that is, when $x \in S_{1}$. Additionally, the condition $I+A \leq \Delta$ is considered. Consequently, the switch from "Deconfinement" to "Free movement" is produced if $x \in S_{1} \bigcap\{x \in \mathscr{X}: I+A \leq \Delta\}$.

\subsection{The multi-phase epidemic model as a hybrid automaton}

The dynamical processes and transitions described in Section 3.1 may be considered as a hybrid dynamical system and be represented by a nonlinear hybrid automaton (Johansson, Egerstedt, Lygeros, and Sastry 
1999, Navarro-López and Carter 2011, Navarro-López and Laila 2013, Navarro-López and Carter 2016). We propose the SEIARD hybrid automaton $\left(H_{S E I A R D}\right)$ based on the defined disease's phases in our epidemic model. The following general hybrid automaton without inputs and outputs, which was proposed in (Navarro-López and Laila 2013, Navarro-López and Carter 2016) will be used as the basic hybrid automaton model.

Definition 1. A hybrid automaton $H$ is a collection $H=(Q, E, \mathscr{X}$, Dom, $\mathscr{F}$, Init, $G, R)$ that models a hybrid dynamical system, where:

- $Q=\left\{q_{1}, q_{2}, \ldots, q_{N_{q}}\right\}$ is a finite set of discrete locations.

- Continuous state-space: $\mathscr{X} \subseteq \mathbb{R}^{n}$.

- Transitions or events: $E \subseteq Q \times Q$ is a finite set of edges.

- Location domains: Dom $: Q \rightarrow 2^{\mathscr{X}}$ is the location domain (sometimes called an invariant). It assigns a set of continuous states to each discrete location $q_{i} \in Q$, thus, $\operatorname{Dom}\left(q_{i}\right) \subseteq \mathscr{X}$.

- Continuous dynamics: $\mathscr{F}=\left\{f_{q_{i}}(x): q_{i} \in Q\right\}$ is a finite set of vector fields describing the continuous dynamics in each location, such that $f_{q_{i}}: \mathscr{X} \rightarrow \mathscr{X}$. Each $f_{q_{i}}(x)$ is assumed to be Lipschitz continuous on the location domain for $q_{i}$ in order to ensure that the solution exists and is unique.

- Set of initial states: $\operatorname{Init} \subseteq \bigcup_{q_{i} \in Q} q_{i} \times \operatorname{Dom}\left(q_{i}\right) \subseteq Q \times \mathscr{X}$.

- Guard maps: $G: E \rightarrow 2^{\mathscr{X}}$. $G$ assigns to each edge a set of continuous states; this set contains the states which enable the edge to be taken.

- Reset maps: $R: E \times \mathscr{X} \rightarrow 2^{\mathscr{X}}$ is a reset map for the continuous states for each edge. For each $e=\left(q_{i}, q_{j}\right) \in E$ and $x \in G(e), R(e, x) \subset \operatorname{Dom}\left(q_{j}\right)$. It is assumed to be non-empty, so that the dynamics can only be changed, not destroyed.

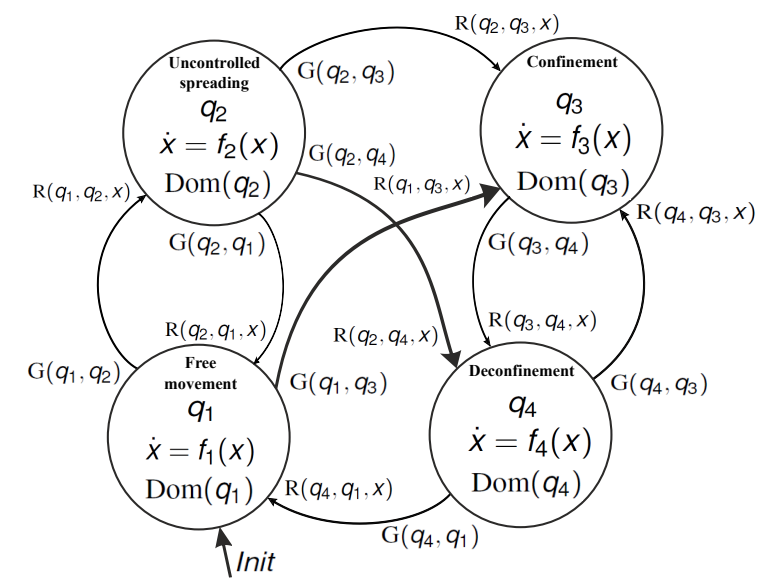

Figure 2: Graphical representation of the SEIARD hybrid automaton $\left(H_{S E I A R D}\right)$ for the multi-phase SEIARD model with one deconfinement phase $\left(f_{i}(x)=f_{q_{i}}(x), \forall i\right)$.

Definition 2. The SEIARD hybrid automaton $H_{S E I A R D}$ with 4 discrete locations describing the dynamics of the multi-phase SEIARD model with one deconfinement phase is a particular case of $H$ with,

- $Q=\left\{q_{1}, q_{2}, q_{3}, q_{4}\right\}$, with $q_{1} \equiv$ "Free movement", $q_{2} \equiv$ "Uncontrolled spreading", $q_{3} \equiv$ "Confinement" and $q_{4} \equiv$ "Deconfinement".

- $\quad \mathscr{X} \subseteq \mathbb{R}^{6}$ as defined in (14), $x=(S, E, I, A, R, D)^{T}$.

- $\operatorname{Dom}\left(q_{1}\right)=\left\{x \in S_{1}\right\}$ with $S_{1}$ as defined in (15), Dom $\left(q_{2}\right)=\left\{x \in S_{2}\right\}$ with $S_{2}$ as defined in (16), $\operatorname{Dom}\left(q_{3}\right)=S_{3} \cup S_{3}^{*} \cup\left\{x \in \mathscr{X}: D>\Delta_{2}\right\}$ with $S_{3}$ and $S_{3}^{*}$ as defined in (19) and (20), respectively, 
and $\operatorname{Dom}\left(q_{4}\right)=S_{4} \bigcap S_{4}^{*} \bigcap\left\{x \in \mathscr{X}: D \leq \Delta_{2}, I+A>\Delta\right\}$ with $S_{4}$ and $S_{4}^{*}$ as defined in (17) and (18), respectively. $\Delta$ and $\Delta_{2}$ are strictly positive constants.

- $\quad$ The dynamics for each discrete location $q_{i}$ are defined in system (1)-(6).

- $G\left(q_{1}, q_{2}\right)=\operatorname{Dom}\left(q_{2}\right), G\left(q_{1}, q_{3}\right)=G\left(q_{2}, q_{3}\right)=\{x \in \mathscr{X}: E>S\}, G\left(q_{2}, q_{1}\right)=\operatorname{Dom}\left(q_{1}\right), G\left(q_{2}, q_{4}\right)=$ $\operatorname{Dom}\left(q_{4}\right), G\left(q_{3}, q_{4}\right)=\operatorname{Dom}\left(q_{4}\right), G\left(q_{4}, q_{3}\right)=\operatorname{Dom}\left(q_{3}\right), G\left(q_{4}, q_{1}\right)=\operatorname{Dom}\left(q_{1}\right) \cap\{x \in \mathscr{X}: I+A \leq \Delta\}$.

- $R\left(q_{\mathrm{i}}, q_{\mathrm{j}}, x\right)=\{x\}, \forall i, j \in\{1, \ldots, 4\}$, and $i \neq j$.

\section{SIMULATION RESULTS}

\subsection{Simulation of the SEIARD model}

Four representative scenarios are shown for the simulation of model (1)-(6) for a generic phase $i$, inspired by real case studies given in (Carcione, Santos, Bagaini, and Ba 2020) and (De la Sen, Agarwal, Nistal, Alonso-Quesada, and Ibeas 2018):

- Scenario 1 shown in Figure 3. We consider a relatively low transmission rate $\beta_{i}=\beta_{A_{i}}=0.3$ day $^{-1}$, a higher proportion of symptomatic infected $p=0.6$, a not very high proportion of susceptible individuals vaccinated $\lambda_{i}=0.35$, and a relatively slow transition from the recovered to the susceptible $\eta=1 / 365$ day $^{-1}$, with an immunity period of 365 days. $R_{0_{i}}=0.0009$ is obtained.

- Scenario 2 shown in Figure 3. We consider a relatively high transmission rate $\beta_{i}=\beta_{A_{i}}=10$ day $^{-1}$ and a higher proportion of symptomatic infected $p=0.6$. We also have a higher proportion of susceptible individuals vaccinated, with half of the susceptible subpopulation vaccinated, $\lambda_{i}=0.5$, and a relatively slow transition from the recovered to the susceptible $\eta=1 / 365 \mathrm{day}^{-1}$, with an immunity period of 365 days. $R_{0_{i}}=0.0588$ is obtained.

- Scenario 3 shown in Figure 4. We consider a relatively high transmission rate $\beta_{i}=\beta_{A_{i}}=10$ day $^{-1}$ and a higher proportion of symptomatic infected $p=0.6$. We also consider that no susceptible individual has been vaccinated, $\lambda_{i}=0$, and a relatively fast transition from the recovered to the susceptible $\eta=1 / 20$ day $^{-1}$, with an immunity period of 20 days. It is clear how the probability to belong to the exposed subpopulation increases and the probability to belong to the recovered subpopulation decreases in comparison to scenarios 1 and 2. $R_{0_{i}}=0.0588$ is obtained.

- Scenario 4 shown in Figure 4 . We consider a relatively high transmission rate $\beta_{i}=\beta_{A_{i}}=10$ day $^{-1}$ and a higher proportion of asymptomatic infected $p=0.1$. Now, all the susceptible individuals have been vaccinated, $\lambda_{i}=1$. We consider a relatively slow transition from the recovered to the susceptible $\eta=1 / 365$ day $^{-1}$, with an immunity period of 365 days. When the response is compared to scenario 3 , it is clear how the increase of the proportion of vaccinated individuals and the increase of the immunity period lead to the decrease of the probability to belong to the exposed subpopulation, the rapid decrease of the probability to belong to the susceptible subpopulation and the increase of the probability to belong to the recovered subpopulation. $R_{0_{i}}=0.0600$ is obtained.

The following parameters are fixed for the four scenarios, with values inspired by real case studies in (Carcione, Santos, Bagaini, and Ba 2020) and (De la Sen, Agarwal, Nistal, Alonso-Quesada, and Ibeas 2018):

$$
\mu=\tau=1 /(10 \cdot 365) \text { day }^{-1}, \gamma=1 / 5 \text { day }^{-1}, \alpha=0.006 \text { day }^{-1}, \varepsilon=1 / 6 \text { day }^{-1} .
$$

For the computation of $R_{0_{i}}$, we use $\zeta_{1}=0.1, \zeta_{2}=0.2$. The initial conditions considered for all the scenarios are $x_{0}=(0.735,0.1,0.08,0.05,0.03,0.005)^{T}$. The simulations have been carried out in OpenModelica. The results obtained are qualitatively in accordance with what you would expect from the dynamical evolution of the probabilities to belong to the different subpopulations considered. It is important to notice how $R_{0_{i}}$ reflects the increase of the transmission rate. We have made extensive simulations with different parameters; for some cases $R_{0_{i}}$ did not give consistent results. The most significant scenarios have been shown. 
(1)

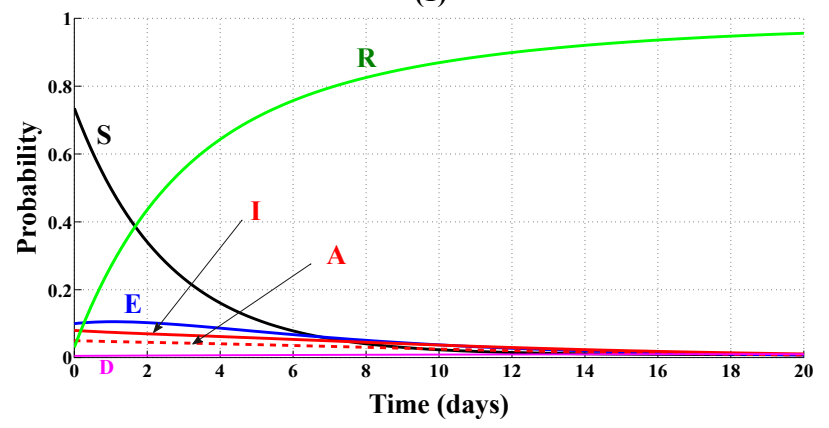

(2)

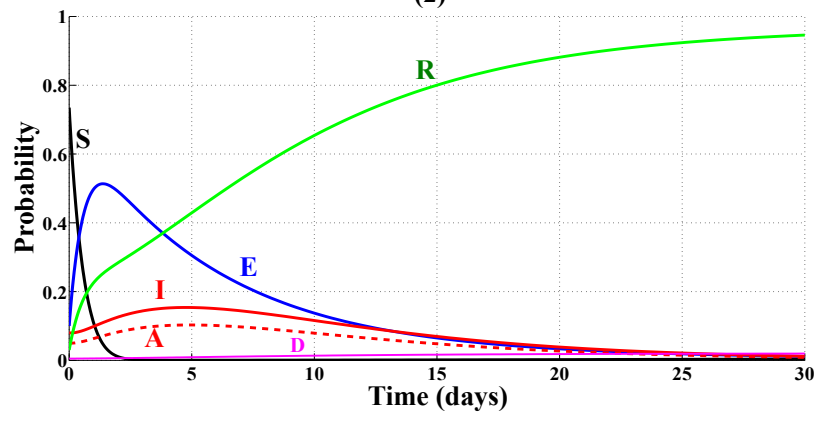

Figure 3: Evolution of the time-dependent probabilities to belong to the $S, E, I, A, R$ and $D$ subpopulations of model (1)-(6) for parameters corresponding to: (1) scenario 1, (2) scenario 2.

Figure 3 shows how, in scenario 1, the probability to belong to the $E$ subpopulation is less than in scenario 2. In scenario 2, at the beginning, $E$ hugely increases to finally evolve close to 0 . Moreover, $I$ and $A$ are higher in scenario 2 than in scenario 1 . In scenario 2, the probability to belong to the $S$ subpopulation decreases faster than in scenario 1 . In Figure 4, it is observed that $E$ is significantly higher (with an initial sharp increase) in scenario 3 than in scenario 4 . For scenarios 3 and $4, A$ and $I$ are swapping their probabilities in time. For scenario 3, $I$ is higher than $A$, and for scenario $4, A$ is higher than $I$.

(1)

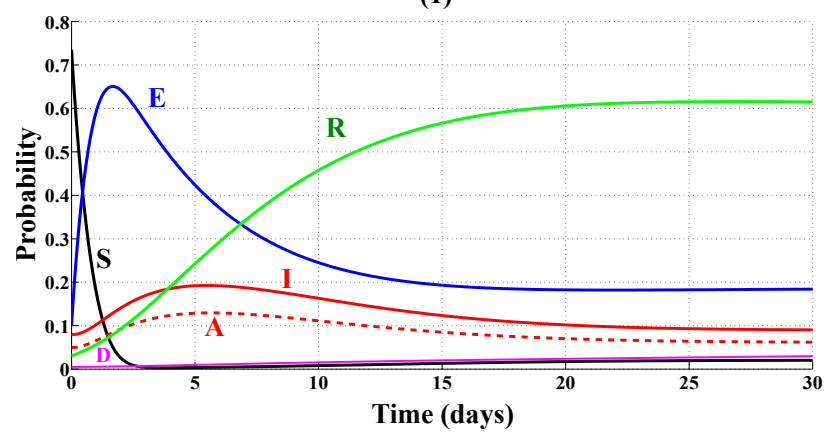

(2)

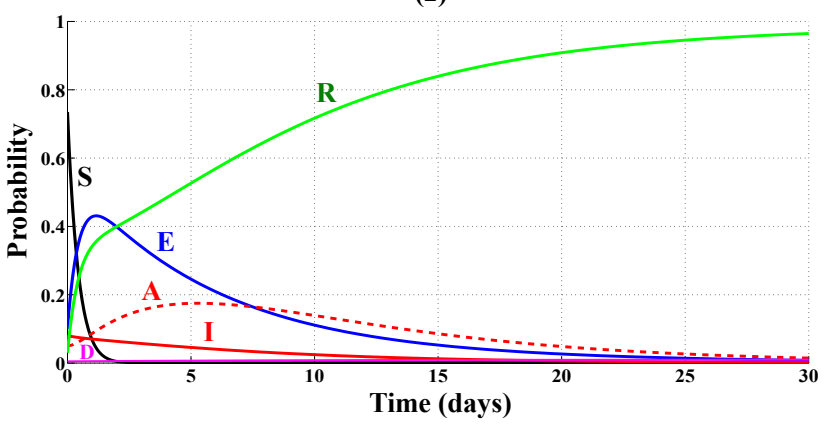

Figure 4: Evolution of the time-dependent probabilities to belong to the $S, E, I, A, R$ and $D$ subpopulations of model (1)-(6) for parameters corresponding to: (1) scenario 3, (2) scenario 4.

\subsection{Simulation of the SEIARD hybrid automaton}

The SEIARD hybrid automaton of Definition 1 is simulated for $q_{0} \times x_{0}=q_{1} \times$ $(0.735,0.1,0.08,0.05,0.03,0.005)^{T}$ by using OpenModelica. The results are discussed below and presented in Figure 5. $R_{0_{i}}$ is the basic reproduction ratio for each phase of the disease $q_{i}$, with $i \in\{1, \ldots, 4\}$, calculated with equation (13). Two different scenarios are reported with switching transmission rate and vaccination strategy:

- Scenario 5. $\eta=1 / 365$ and: $\beta_{1}=0.8, \lambda_{1}=0\left(R_{0_{1}}=0.0025\right) ; \beta_{2}=2, \lambda_{2}=0.35\left(R_{0_{2}}=0.0070\right)$; $\beta_{3}=12, \lambda_{3}=1\left(R_{0_{3}}=0.0785\right) ; \beta_{4}=1, \lambda_{4}=0.6\left(R_{0_{4}}=0.0032\right)$. The vaccination strategy works only for some months and the system evolves finally to "Confinement" $\left(q_{3}\right)$.

- Scenario 6. $\eta=1 / 365$ and: $\beta_{1}=0.8, \lambda_{1}=0.2\left(R_{0_{1}}=0.0025\right) ; \beta_{2}=2, \lambda_{2}=0.8\left(R_{0_{2}}=0.0070\right)$; $\beta_{3}=12, \lambda_{3}=0.8\left(R_{0_{3}}=0.0785\right) ; \beta_{4}=1, \lambda_{4}=0.8\left(R_{0_{4}}=0.0032\right)$. Comparing to scenario 5 , in 
scenario 6 , the vaccination strategy is changed to increase the proportion of vaccinated susceptible individuals. This makes the system finally evolve to "Free movement" $\left(q_{1}\right)$.

For the computation of $R_{0_{i}}$, we use $\zeta_{1}=0.1, \zeta_{2}=0.2$. The rest of the parameters for both scenarios are:

$\mu=\tau=1 /(10 \cdot 365)$ day $^{-1}, \gamma=1 / 5$ day $^{-1}, \alpha=0.006$ day $^{-1}, \varepsilon=1 / 6$ day $^{-1}, p=0.6, \Delta=0.1, \Delta_{2}=0.03$.

(1)
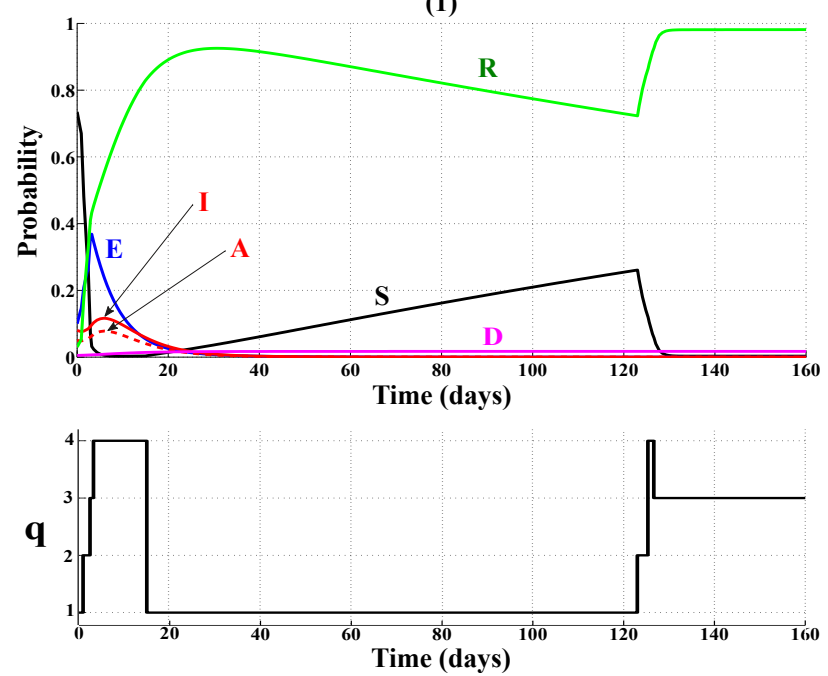

(2)
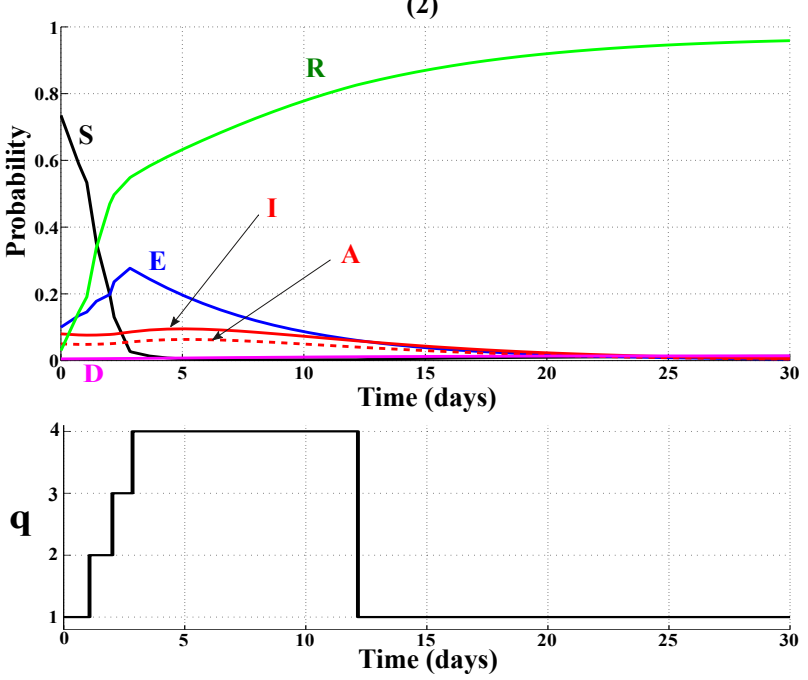

Figure 5: Simulation of the SEIARD hybrid automaton corresponding to: (1) scenario 5, (2) scenario 6.

It is important to highlight the qualitative meaning of the parameters used and the results obtained. Their relevance is not in their specific numeric value but in their relationships with other parameters and their influence on the dynamics of the evolution of the disease.

\section{CONCLUSIONS}

We have successfully reproduced the evolution over time of the phases of an infectious disease. This has been possible by the proposal of the SEIARD hybrid automaton. The proposed hybrid automaton is especially effective in the specification of the transitions between the different phases of the system, which becomes critical when multiple transitions depending on the system dynamics are present. Our novel contribution is not only in the proposal of a framework to simulate multi-phase epidemic models, but also in the proposal of an extended version of the SEIARD epidemic model that unifies the distinction between symptomatic and asymptomatic infectious subpopulations, the consideration of an immunity period, the influence of vaccination in the evolution of the disease, and the changes of the transmission rate, and consequently the changes of the basic reproduction ratio, depending on the evolution of the disease. The use of a computational-dynamical framework for specifying the dynamical properties of the system can facilitate the design of control algorithms for more complex epidemic models.

\section{REFERENCES}

Berman, A., and R. Plemmons. 1994. Nonnegative matrices in the mathematical sciences. Society for Industrial and Applied Mathematics.

Carcione, J. M., J. E. Santos, C. Bagaini, and J. Ba. 2020. "A simulation of a COVID-19 epidemic based on a deterministic SEIR model". Frontiers in Public Health vol. 8, pp. 1-13. 
Chladná, Z., J. Kopfová, D. Rachinskii, and S. C. Rouf. 2020. "Global dynamics of SIR model with switched transmission rate". Mathematical Biology vol. 80, pp. 1209-1233.

De la Sen, M., R. P. Agarwal, R. Nistal, S. Alonso-Quesada, and A. Ibeas. 2018. "A switched multicontroller for an SEIADR epidemic model with monitored equilibrium points and supervised transients and vaccination costs". Advances in Difference Equations vol. 390, pp. 1-31.

De la Sen, M., A. Ibeas, S. Alonso-Quesada, and R. Nistal. 2017. "On a new epidemic model with asymptomatic and dead-infective subpopulations with feedback controls useful for ebola disease". Discrete Dynamics in Nature and Society vol. 2017, pp. 1-22.

den Driessche, P. V., and J. Watmough. 2002. "Reproduction numbers and sub-threshold endemic equilibria for compartmental models of disease transmission". Mathematical epidemiology vol. 180, pp. 29-48.

Diekmann, O., J. Heesterbeek, and J. Metz. 1990. "On the definition and the computation of the basic reproduction ratio R 0 in models for infectious diseases in heterogeneous populations". Journal of Mathematical biology vol. 28, pp. 365-382.

Graphext 2020-2021. "Fighting Coronavirus with Tech \& Data". bit.ly/corona-data.

Johansson, K., M. Egerstedt, J. Lygeros, and S. Sastry. 1999. "On the regularization of Zeno hybrid automata". Systems \& Control Letters vol. 38 (3), pp. 141-150.

Liu, X., and P. Stechlinski. 2017. "The switched SIR model”. In Infectious Disease Modeling. A Hybrid System Approach, pp. 43-82. Switzerland, Springer.

Meng, X., and L. Chen. 2008. "The dynamics of a new SIR epidemic model concerning pulse vaccination strategy". Applied Mathematics and Computation vol. 197, pp. 582-597.

Navarro-López, E., and R. Carter. 2011. "Hybrid automata: an insight into the discrete abstraction of discontinuous systems". International Journal of Systems Science vol. 42 (11), pp. 1883-1898.

Navarro-López, E., and R. Carter. 2016. "Deadness and how to disprove liveness in hybrid dynamical systems". Theoretical Computer Science vol. 642, pp. 1-23.

Navarro-López, E. M., and D. S. Laila. 2013. "Group and total dissipativity and stability of multi-equilibria hybrid automata". IEEE Transactions on Automatic Control vol. 58 (12), pp. 3196-3202.

Pröll, S. 2013. Stability of Switched Epidemiological Models. MSc thesis, Julius-Maximilians-Universität Würzburg Institut für Mathematik, Würzburg, Germany. Available via https://core.ac.uk/download/pdf/ 35101342.pdf. Accessed March 1, 2021.

van den Driessche, P. 2017. "Reproduction numbers of infectious disease models". Infectious Disease Modelling vol. 2, pp. 288-303.

\section{AUTHOR BIOGRAPHIES}

EVA M. NAVARRO-LÓPEZ is a Reader (Associate Professor) in Data Science at the University of Wolverhampton. She holds a $\mathrm{PhD}$ in Automatic Control and Industrial Electronics from Universitat Politècnica de Catalunya and an MEng in Computer Science Engineering, a BSc in Physical Systems Engineering and a BEng in Systems and Electronics from Universitat d'Alacant/Universidad de Alicante. Her research interests lie in computer science, control engineering, mathematical systems analysis and modelling, systems biology, computational neuroscience, artificial intelligence, automated formal verification and network science. Her email address is E.NavarroLopez@wlv.ac.uk.

NURDAN ÇABUKOĞLU is a Lecturer (Assistant Professor) at the University of Wolverhampton. She holds a PhD in Mathematical Biology and Ecology from the University of Leicester. Her research interests include animal movement, diffusion-reaction equations, population dynamics and infectious disease modelling. Her email address is N.Cabukoglu@wlv.ac.uk. 